\title{
Características Biométricas e Hemodinâmica da Artéria Supra Testicular em Machos Brangus de 12 Meses de Idade
}

\section{Biometric and Hemodynamic Characteristics of the Supra Testicular Artery in 12-Month-Old Brangus Males}

\author{
Fábio Procópio Ribeiroa; Patrícia da Cruz Favaroa; Victor Hugo Gonçalves Galdiolia; Gabriel Ribas Pereira ; \\ Ivis da Silva Dias ${ }^{\mathrm{b}}$; Flávio Guiselli Lopesa; Celso Koetz Junior ${ }^{\mathrm{a}}$; Flávio Antônio Barca Junior ${ }^{\mathrm{b}}$ \\ aUnopar, Programa de Pós-Graduação Stricto Sensu em Saúde e Produção Animal. PR, Brasil. \\ bUnopar. PR, Brasil. \\ *E-mail: fabio_procopio7@hotmail.com
}

\begin{abstract}
Resumo
Parâmetros biométricos, como formato testicular, espessura de pele, são importantes na avaliação da hemodinâmica testicular e, pode contribuir para compreensão dos mecanismos termorreguladores em animais domésticos. O objetivo deste estudo foi avaliar as características biométricas em relação a hemodinâmica testicular utilizando a ultrassonografia Doppler. Foram avaliados 52 machos de 12 meses de idade da raça Brangus (3/8 Brahman x 5/8 Aberdeen Angus) por meio de parâmetros de velocidade média (VM), velocidade sistólica (VP), velocidade diastólica (VE), índice de pulsatilidade (IP) e índice de resistência (IR), usando ultrassonografia Doppler. Os procedimentos foram aprovados pelo Comitê de Ética para Cuidados e Uso de Animais Experimentais da Universidade Norte do Paraná (CEUAs/UNOPAR no. 010/14). Os dados do índice de temperatura e umidade (ITU) foram coletados de uma estação meteorológica automática TGD-400 (Instrutherm Inc.). Os dados foram analisados utilizando análise de variância (ANOVA). Foi utilizado teste de Tukey e correlação de Pearson com nível de significância de $5 \%$. O ITU foi de 74,5 durante o experimento. A média do perímetro escrotal (PE) e espessura de pele testicular foram de $32,86 \pm 2,25 \mathrm{~cm}$ e 4,77 $\pm 0,87 \mathrm{~cm}$, respectivamente. As classificações de formato moderado/oval (MO; $\mathrm{n}=12)$, a espessura de pele foi maior $(5,21 \pm 1,05 ; \mathrm{P}<$ 0,05), comparado com longo/moderado (LM; $n=40) ;(4,64 \pm 0,80)$. O ultrassom Doppler permitiu avaliar as alterações do fluxo sanguíneo da artéria supra testicular sugerindo que as diferenças na espessura da pele escrotal estejam relacionadas com os requisitos fisiológicos para o desempenho reprodutivo em bovinos da raça Brangus.
\end{abstract}

Palavras chave: Bovinos. Termorregulação. Ultrassonografia Doppler. Índice de Temperatura e Umidade.

\begin{abstract}
Biometric parameters, such as testicular shape, skin thickness, are important in the testicular hemodynamics evaluation and can contribute to the understanding of thermoregulatory mechanisms in domestic animals. The aim of this study was to evaluate the biometric characteristics in relation to testicular hemodynamics using Doppler ultrasonography. 52 12-month-old males were evaluated, breed Brangus (3/8 Brahman $x$ 5/8 Aberdeen Angus) using parameters of mean speed (MV), systolic speed (PV), diastolic speed (VE), pulsatility index (IP) and resistance index (IR), using Doppler ultrasonography. The procedures were approved by the Ethics Committee for the Care and Use of Experimental Animals at University Northern Paraná (CEUA / UNOPAR no. 010/14). The temperature and humidity index (ITU) data were collected from an automatic meteorological station TGD-400 (Instrutherm Inc.). The data were analyzed using analysis of variance (ANOVA). Tukey test and Pearson correlation were used with a 5\% significance level. ITU was 74.5 during the experiment. The average scrotal circumference (PE) and testicular skin thickness were $32.86 \pm 2.25 \mathrm{~cm}$ and $4.77 \pm 0.87 \mathrm{~cm}$, respectively. The classifications of moderate / oval shape (OM; $n=12)$, the skin thickness was greater (5.21 $\pm 1.05 ; P<0.05)$, compared to long / moderate (LM; $n=40) ;(4.64 \pm 0.80)$. Doppler ultrasound allowed the assessment of changes in the blood flow of the supra testicular artery, suggesting that the differences in scrotal skin thickness are related to the physiological requirements for reproductive performance in Brangus cattle.
\end{abstract}

Keywords: Cattle. Thermoregulation. Doppler Ultrasound. Temperature and Humidity Index.

\section{Introdução}

As variações ambientais extremas interferem na fertilidade dos touros e causam prejuízos para a eficiência reprodutiva do rebanho. Portanto, as condições ambientais são determinadas por meio do índice de conforto térmico gerado através da avaliação de temperatura e umidade (LI et al., 2009). O Índice de Temperatura e Umidade (ITU) é um excelente indicador muito utilizado para a avaliação do efeito de alterações climáticas na produção de leite (AZEVEDO et al., 2005; LI et al., 2009). Índices superiores a 72 caracterizam um ambiente estressante para bovinos de leite (RAVAGNOLO et al., 2000), porém poucos estudos avaliaram qualidade seminal de touros durante a estação de monta em situações de elevados ITU
(RAVAGNOLO et al., 2000; MENEGASSI et al., 2014; 2016). Temperaturas mais elevadas podem afetar negativamente o potencial reprodutivo de mamíferos gerando um estresse térmico, podendo até chegar à infertilidade (WOLFENSON; ROTH, 2019). O estresse térmico prejudica não somente a normalidade da função testicular, mas também afeta negativamente a capacidade fecundante dos espermatozoides produzidos e põe em risco o desenvolvimento de embriões gerados a partir destes gametas.

Nos animais domésticos, a artéria testicular é uma estrutura envolvida em forma de cone dispostas na porção cranial ou dorsal do testículo. Esses envoltos entre artéria e veias é denominado plexo pampiniforme, que através de mecanismo 
contracorrente, o sangue arterial é resfriado pelo sangue venoso. $\mathrm{O}$ escroto, testículos e a vascularização testicular têm papel extremamente importante na manutenção da temperatura testicular, mantendo-os de 2 a $6^{\circ} \mathrm{C}$ abaixo da temperatura do corporal, o que é essencial para a espermatogênese normal no touro (KASTELIC et al., 1997; BRITO et al., 2004; KASTELIC, 2014). Animais que apresentam maior perímetro escrotal (PE) possuem fenotipicamente e geneticamente características reprodutivas, que são transmitidas a ambos os sexos (COULTER; FOOTE, 1979; SMITH et al., 1989; CORBET et al. 2013). Além disso, o PE pode ser utilizado como um indicador de puberdade do touro (WILDEUS et al., 1984).

A ultrassonografia (US) Doppler é um método não invasivo que permite avaliar a vascularização de vários órgãos de animais domésticos, inclusive órgão reprodutivo com intuito de identificar anormalidades testiculares (MATTON; NYLAND, 2004; POZOR; MCDONNEL, 2004; CARRILLO et al., 2012; de BRITO et al., 2015). Esta técnica é utilizada largamente para identificar infertilidade em humanos (PINGGERA, 2008). Em bovinos, a técnica por US Doppler é utilizada em fêmeas, para a avaliação do fluxo sanguíneo uterino, ovariano e implantação embrionária (HERZOG; BOLLWEIN, 2007; PUGLIESI et al., 2019). A avaliação por ultrassonografia Doppler do conteúdo escrotal representa uma das técnicas atuais que podem ser exploradas para avaliar a função e a perfusão vascular em reprodutores bovinos (BARCA JUNIOR et al., 2018; CLAUS et al., 2019).

O presente estudo teve como objetivo, avaliar a biometria e a hemodinâmica testicular com a utilização da técnica de US Doppler em machos da raça Brangus.

\section{Desenvolvimento}

Todos os procedimentos utilizados foram aprovados pelo Comitê de Ética para Cuidados e Uso de Animais Experimentais da Universidade Norte do Paraná (CEUA/ UNOPAR $\left.n^{\mathbf{o}} 010 / 14\right)$.

\subsection{Local e animais}

O experimento foi realizado em uma propriedade localizada no município de Mandaguari, Estado do Paraná com latitude $23^{\circ} 32^{\prime} 52^{\prime}$ S e longitude $51^{\circ} 40^{\prime} 15^{\prime} \mathrm{O}$, com altitude média de 670 metros. O clima do município, segundo a classificação de Köppen, é considerado $\mathrm{Cfb}$ (temperado úmido com verão temperado) (IBGE, 2016). Foram utilizados 52 machos hígidos, da raça Brangus (3/8 Brahman x 5/8 Aberdeen Angus) e idade média de 12 meses. Os animais foram mantidos em regime extensivo de criação, permanecendo neste sistema até o período da desmama. Após a desmama, aos sete meses, os animais foram destinados ao sistema intensivo de criação, em barracão coberto com $780 \mathrm{~m}^{2}$ e $36,4 \mathrm{~m}$ de cocho sendo $70 \mathrm{~cm}$ por animal, água a vontade.

Receberam, durante cinco meses, silagem de milho e mistura concentrada com 18\% de PB recebendo $1,5 \%$ do Peso Vivo. Os animais foram suplementados com sal mineral e água ad libitum. Os animais foram avaliados em julho de 2019 e permanecem no mesmo sistema para serem utilizados como reprodutores ou para engorda de acordo com as necessidades do criador.

\section{2 Índice de temperatura e umidade}

A avaliação dos parâmetros bioclimáticos foi realizada através da estação meteorológica automática TGD-400 (Instrutherm Inc.), que possui um sensor de bulbo seco que possibilita a medição da temperatura ambiental, um sensor de bulbo úmido que afere a taxa de evaporação indicando os efeitos da umidade no indivíduo, e o globo térmico que realiza indicação da exposição ao calor do indivíduo em razão da luz direta e de outros objetos radiantes de calor no ambiente (INSTRUTHERM, 2010).

Os dados de temperatura e umidade do ar foram coletados com intervalos de 15 minutos entra as coletas das amostras até o final dos procedimentos (14:00 às 19:00). O ITU foi calculado conforme a fórmula:

$$
\mathrm{ITU}=\{(0.8 \mathrm{~T})+[(\mathrm{UR} / 100) \times(\mathrm{Tdb}-14.4)]+46.4\}
$$

Sendo que $\mathrm{T}$ é a temperatura do ar em ${ }^{\circ} \mathrm{C}$, Tdb é a temperatura do ar medida por termômetro de bulbo seco $\left({ }^{\circ} \mathrm{C}\right)$ e UR é a umidade relativa (\%) (THOM, 1959).

\subsection{Avaliação da biometria testicular}

As medidas de perímetro escrotal foram realizadas em tronco próprio de contenção, mantidos em posição quadrupedal e sem a utilização de sedação. Realizou-se a medição da parte de maior diâmetro do escroto, utilizando fita métrica metálica do tipo scrotal tape (Nasco Inc.). As medições da biometria testicular foram: comprimento do testículo direito, comprimento do testículo esquerdo, largura do testículo direito e largura do testículo esquerdo, foram feitas com auxílio de um paquímetro, em que foi considerada apenas a porção glandular do testículo, excluindo a cauda do epidídimo.

As medidas de espessura da pele foram tomadas em tronco de contenção e feitas com o ultrassom operando em modo-B, em que o transdutor foi posicionado sempre sobre o testículo esquerdo, na posição ventro-dorsal, sob o eixo longitudinal e utilizado o mediastino testicular como ponto de referência.

Para o cálculo do volume se adotou a fórmula do cilindro (FIELDS et al., 1979), em que:

\section{Volume $=2\left[\left(\mathrm{r}^{2}\right) \times \pi \times \mathrm{h}\right]$}

Em que: $r=$ raio calculado a partir da largura (largura/2), $\mathrm{h}=$ comprimento ou altura, e $\pi(\mathrm{Pi})=3,14$.

Para a determinação da forma testicular se utilizou metodologia que classifica o formato de acordo com a razão da largura/comprimento (BAILEY et al.,1996; 1998), em que 1 significa largura igual a $1 / 2$ do comprimento e 5 significa largura igual ao comprimento. Em função desta escala foram estabelecidas as seguintes formas:

- Longo (L) em que a razão foi $\leq 0,5$;

- Longo/Moderado (L/M) em que a razão foi > 0,5 a 0,625; 
- Longo/Oval (L/O) em que a razão foi de 0,626 a 0,750;

- Moderado/Oval (M/O) em que a razão foi de 0,751 a 0,875 ;

- Esférico (E) em que a razão foi $>0,875$.

\subsection{Avaliação da hemodinâmica pela técnica de ultrassonografia Doppler}

As imagens Doppler foram realizadas utilizando um ultrassom portátil com um transdutor linear de 7,5 MHz (L741V), modelo (SonoScape A6V, DOMED, Valinhos, Brasil). As avaliações foram realizadas sempre por uma equipe de dois operadores, o primeiro para a realização da insonação e o segundo operando o equipamento de ultrassom. $\mathrm{O}$ transdutor foi posicionado distalmente ao cordão espermático e junto ao parênquima testicular, definindo assim uma posição anatômica fixa para todos os animais, permitindo ainda obter uma posição perpendicular em relação à artéria supra testicular com o intuito de obter ecos mais claros.

As aferições ocorreram sempre na maior porção do vaso arterial visualizado junto ao plexo pampiniforme, em todas as medidas realizadas o ângulo de insonação do Doppler e o eixo longitudinal do vaso nunca foi superior a $60^{\circ}$, evitando assim subestimação do pico sistólico (BARCA JUNIOR et al., 2018; GERHARD-HERMAN et al., 2006). Durante a realização da avaliação foi aplicado gel sobre a superfície da pele no local determinado e com a mínima pressão necessária teve início a varredura no modo $\mathrm{B}$ e, assim que identificada a artéria supra testicular foi ativado o sistema colorido Doppler. Após localizada a artéria supra testicular, o sistema de Color Doppler e o modo PW (Pulsed Wave) foram iniciados, para captar os ciclos cardíacos do animal, sendo a imagem fixada, podendo assim, ser feita a medição de um ciclo para obter os parâmetros da velocidade em sístole (VP), velocidade em diástole (VE), velocidade média (VM), índice de pulsatilidade (IP) e índice de resistência (IR). Imagem ultrassonográfica em modo colorido Doppler da artéria supra testicular apresentando padrão de ondas monofásico do fluxo sanguíneo ilustrada na Figura 1.

Figura 1 - Imagem ultrassonográfica em modo colorido Doppler da artéria supra testicular apresentando padrão de ondas monofásico do fluxo sanguíneo em um macho Brangus criado em confinamento

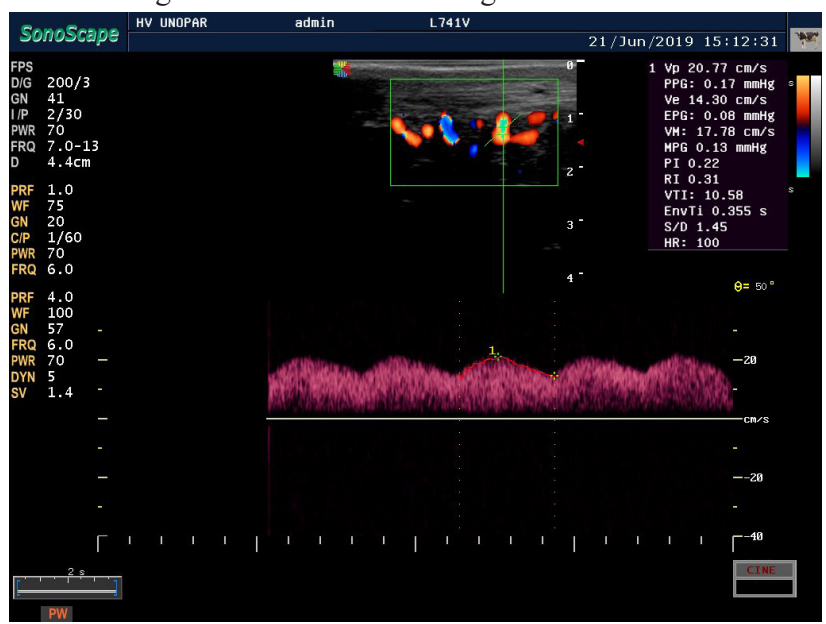

Fonte: Dados da pesquisa.
As informações registradas foram a VP, VE, e VM (cm/s), o IP e o IR. O IP foi determinado pela velocidade máxima do fluxo sanguíneo menos a velocidade mínima, este resultado foi dividido pela velocidade média. Já o IR foi obtido através da subtração da velocidade no pico sistólico da velocidade final da diástole, valor este que foi dividido pela velocidade do pico de sístole (MIDDLETON et al., 1989), cujos valores já eram determinados e apresentados pelo equipamento utilizado.

\subsection{Análise estatística}

Os dados coletados foram apresentados por estatística descritiva, na forma de média e desvio padrão, para a comparação entre os diferentes formatos testicular foi utilizada a análise de variância (ANOVA), após a verificação da normalidade dos dados. Como teste de média foi utilizado teste de Tukey. Foi estabelecida a correlação de Pearson para as variáveis biométricas e hemodinâmicas mensuradas. O nível mínimo de significância utilizado foi de $5 \%$ e os cálculos foram realizados com o auxílio do pacote estatístico $\mathrm{R}(\mathrm{R}$ CORE TEAM, 2014).

\subsection{Resultados e Discussão}

A temperatura média nos dias das medições foi de 27,57 $\pm 0,49^{\circ} \mathrm{C}$ e a umidade relativa de $60,1 \pm 4,5 \%$, sendo o ITU médio de 74,5 durante a realização do experimento. Os animais apresentaram peso médio de 414,02 $\pm 25,06 \mathrm{~kg}$ e $382,04 \pm 64,18$ dias de idade.

A média geral do PE e espessura de pele testicular foram de $32,86 \pm 2,25 \mathrm{~cm} \mathrm{e} 4,77 \pm 0,87 \mathrm{~cm}$, respectivamente. $\mathrm{O}$ volume testicular médio do grupo de animais foi 411,05 \pm $91,17 \mathrm{ml}$. As características biométricas do grupo de animais avaliados estão descritas no Quadro 1.

Quadro 1 - Média e desvio padrão das características biométricas de machos da raça Brangus aos 12 meses de idade criados em confinamento

\begin{tabular}{|l|c|c|c|c|c|c|}
\hline $\begin{array}{c}\text { Idade } \\
\text { (meses) }\end{array}$ & $\begin{array}{c}\text { Animais } \\
\text { (n) }\end{array}$ & $\begin{array}{c}\mathbf{P E} \\
\mathbf{( c m )}\end{array}$ & $\begin{array}{c}\text { Compri- } \\
\text { mento } \\
(\mathbf{c m})\end{array}$ & $\begin{array}{c}\text { Largura } \\
\mathbf{( c m})\end{array}$ & $\begin{array}{c}\text { Volume } \\
(\mathbf{m l})\end{array}$ & $\begin{array}{c}\text { Espessura } \\
\text { da Pele } \\
(\mathbf{c m})\end{array}$ \\
\hline 12 & 52 & $\begin{array}{c}32,86 \\
\pm\end{array}$ & $\begin{array}{c}10,08 \pm \\
0,87\end{array}$ & $\begin{array}{c}6,03 \pm \\
0,49\end{array}$ & $\begin{array}{c}411,05 \\
\pm\end{array}$ & $\begin{array}{c}4,77 \pm \\
0,88\end{array}$ \\
\hline
\end{tabular}

PE: Perímetro Escrotal.

Fonte: Dados da pesquisa.

As duas classificações de formato obtidas foram moderado/ oval (MO) com 12 animais e longo/moderado (LM) com 40. A média da espessura de pele dos animais $\operatorname{LM}(4,64 \pm 0,80)$ foi menor do que os animais MO $(5,21 \pm 1,05 ; \mathrm{P}<0,05)$. O PE e o volume testicular foram nos animais com formato testicular $\operatorname{LM}\left(32,98 \pm 2,22 \mathrm{~cm}\right.$ e $\left.412,46 \pm 86,73 \mathrm{~cm}^{3}\right)$ comparados com MO $\left(32,46 \pm 2,39 \mathrm{~cm}\right.$ e $\left.406,38 \pm 108,81 \mathrm{~cm}^{3}\right)$, porém não foram diferentes estatisticamente $(\mathrm{P}>0,05)$. Não foi observada a diferença significativa entre os valores de IR e IP nos dois grupos de formatos avaliados, sendo observado valores menores numericamente nos animais de LM comparados com MO. Os 
resultados do formato testicular em relação à hemodinâmica da artéria supra testicular estão expostos no Quadro 2.

Quando 2 - Média e desvio padrão do formato do testículo em relação às características hemodinâmicas da artéria supra testicular de machos da raça Brangus aos 12 meses de idade criados em confinamento.

\begin{tabular}{|l|c|c|c|c|c|c|c|c|c|}
\hline Formato & $\mathbf{N}$ & $\begin{array}{c}\mathbf{P E} \\
\mathbf{( c m )}\end{array}$ & $\begin{array}{c}\text { Volume } \\
(\mathbf{m l})\end{array}$ & $\begin{array}{c}\text { Espessura } \\
\mathbf{d a} \text { Pele } \\
(\mathbf{m m})\end{array}$ & $\begin{array}{c}\mathbf{V P} \\
(\mathbf{c m} / \mathbf{s})\end{array}$ & $\begin{array}{c}\mathbf{V E} \\
(\mathbf{c m} / \mathbf{s})\end{array}$ & $\begin{array}{c}\text { VM } \\
(\mathbf{c m} / \mathbf{s})\end{array}$ & IP & IR \\
\hline $\mathrm{LM}$ & 40 & $32,98 \pm 2,22^{\mathrm{a}}$ & $412,46 \pm 86,73^{\mathrm{a}}$ & $4,64 \pm 0,80^{\mathrm{a}}$ & $22,88 \pm 5,40^{\mathrm{a}}$ & $10,82 \pm 4,69^{\mathrm{a}}$ & $17,09 \pm 3,76^{\mathrm{a}}$ & $0,48 \pm 0,27^{\mathrm{a}}$ & $0,51 \pm 0,21^{\mathrm{a}}$ \\
\hline $\mathrm{MO}$ & 12 & $32,46 \pm 2,39^{\mathrm{a}}$ & $406,38 \pm 108,81^{\mathrm{a}}$ & $5,21 \pm 1,05^{\mathrm{b}}$ & $22,45 \pm 7,58^{\mathrm{a}}$ & $10,39 \pm 3,99^{\mathrm{a}}$ & $16,53 \pm 4,96^{\mathrm{a}}$ & $0,50 \pm 0,25^{\mathrm{a}}$ & $0,52 \pm 0,16^{\mathrm{a}}$ \\
\hline
\end{tabular}

LM: longo/moderado, MO: moderado oval, VP: Velocidade na sístole, VE: Velocidade em diástole, VM: Velocidade média, IP: Indice de pulsatilidade,

IR: Índice de resistividade, VM: Velocidade média. Letras diferentes na mesma coluna diferem entre si $(\mathrm{p}<0,05)$.

Fonte: Dados da pesquisa.

Foi observado uma correlação positiva entre o PE e volume testicular $(0,839)$. As correlações encontradas entre os valores obtidos da hemodinâmica testicular foram: IP e IR $(0,948)$, VE e IR (-0,794), e VE e IP (-0,750). A VM apresentou uma correlação positiva com a velocidade na sístole $(0,825)$ e velocidade na diástole $(0,695)$. A correlação da espessura da pele com o IR foi de $-0,276$. Os valores dessas correlações encontradas estão dispostos no Quadro 3.

Quadro 3 - Coeficiente de correlação de Pearson (r) entre as variáveis da US Doppler e as características da biometria testicular de machos da raça Brangus de 12 meses de idade criados em confinamento.

\begin{tabular}{|l|c|c|c|c|c|c|c|}
\hline & PE & Volume & Pele & VP & VE & VM & IP \\
\hline Volume & $0,839^{*}$ & & & & & & \\
\hline Pele & NS & NS & & & & & \\
\hline VP & $0,300^{* *}$ & $0,274^{*}$ & NS & & & & \\
\hline VE & NS & NS & NS & NS & & & \\
\hline VM & NS & $0,331^{* *}$ & NS & $0,825^{*}$ & $0,695^{*}$ & & \\
\hline IP & NS & NS & NS & $0,363^{* *}$ & $-0,750^{*}$ & NS & \\
\hline IR & NS & NS & $-0,276^{* *}$ & $0,342^{* *}$ & $-0,794^{*}$ & NS & $0,948^{*}$ \\
\hline
\end{tabular}

PE: Perímetro escrotal, VP: Velocidade na sístole, VE: Velocidade em diástole, VM: Velocidade média, IP: Índice de pulsatilidade, IR: Índice de resistividade, NS: Não significativo, $* \mathrm{P}<0,01,{ }^{* *} \mathrm{P}<0,05$.

Fonte: Dados da pesquisa.

No presente estudo, o ITU obtido foi considerado abaixo da temperatura que prejudica o desequilíbrio fisiológico em função do estresse térmico em animais e, portanto, é provável que nem a temperatura nem a umidade fossem altas o suficiente para causar danos testiculares, que podem levar a consequências diretas na forma testicular (KASTELIC et al.,1997). A raça Brangus é uma mistura de Bos taurus taurus e Bos taurus indicus, mostrando uma adaptação significativa às regiões tropicais e subtropicais, e apresentando maior potencial genético para melhorar a eficiência da produtividade (GALINA et al., 2007). Essas características são muito importantes para o desenvolvimento contínuo da produção de gado de corte no Brasil.

O tamanho e o formato dos testículos são determinados por mecanismos genéticos e efeitos ambientais, de modo que os touros B. taurus indicus possuem testículos mais longos e pele escrotal mais fina comparados aos touros taurinos (DOWLING, 1964; SIQUEIRA et al., 2012). Claus et al., (2018), sugerem que o formato testicular LM e MO não está relacionado à espessura da pele ou à hemodinâmica da artéria supra testicular em animais da raça Braford. Diferentemente, os resultados deste estudo demonstraram menor espessura de pele escrotal em animais com formato LM comparados com MO. Similarmente, os resultados sugeriram que os machos Brangus, com formato LM, têm uma espessura de pele testicular menor sendo responsável para manter a termorregulação adequada. Esses fatores contribuem para uma melhor taxa de troca de calor, com uma maior área de troca e uma barreira física mais fina (BRITO et al., 2003; 2004).

Claus et al. (2018) avaliaram a espessura da pele escrotal de touros Braford aos 16 meses de idade e observaram uma variação de 2,29 a 5,3 mm, além de observarem valores maiores de IR e IP comparados com a média de touros de outras raças, conforme relatado por Junior et al. (2018). Em contraste, estes resultados em Brangus pré-púberes demostraram um maior IR e PI em comparação com animais de idades superiores. A espessura da pele e o peso total da pele aumentam com a idade (DOWLING, 1964) e tanto uma pele fina quanto uma mais grossa podem estar associadas com qualquer estágio de maturidade, bem como sofrerem alterações sazonais nas quais ocorram mudanças na alimentação (DOWLING, 1964; SANYAL; MAJI, 2001). A diferença na espessura da pele também é encontrada entre zebuínos, taurinos e suas cruzas (JIAN et al., 2014). Quando comparado a touros de diferentes raças, o $B$. taurus indicus tem um número aumentado de glândulas sudoríparas na pele do escroto de diferentes tamanhos, além de uma artéria testicular mais longa em relação ao tamanho do testículo, fazendo com que eles dissipem o calor mais facilmente através da transpiração (BRITO et al., 2003). Essas características permitem melhor dissipação de calor e requerem menos sangue em comparação com o gado B. taurus taurus (JIAN et al., 2014).

No presente estudo, os animais apresentaram menor valor de volume para os animais com formatos LM comparados com formato MO. Por outro lado, touros Nelore com idade entre 17 e 20 meses tiveram predominância da forma oval longa, seguida pela forma moderada longa, o que indica uma mudança na forma testicular à medida que a idade avança, resultando em uma forma testicular mais arredondada (VIU et al., 2015). Assim, essas formas com maior área de superfície terão temperaturas mais baixas, porque podem dissipar o calor mais facilmente, ao contrário dos testículos com menor área 
de superfície.

Observou-se alta correlação entre o $\mathrm{PE}$, o volume e a velocidade da sístole nos animais do estudo. Isto se deve a uma redistribuição sistêmica do fluxo para regular a temperatura corporal, que leva a um aumento do aporte sanguíneo destinado ao fluxo testicular. Zelli et al., (2013) observaram em cães que estágios iniciais da espermatogênese são sensíveis à redução no fluxo sanguíneo, em função do defeito energético nas mitocôndrias, justificando os animais que apresentaram alterações seminais. Adicionalmente, Barca Jr et al., (2018) observaram que a VM se apresentou maior em animais da raça Brangus e o menor valor para os animais Nelore, sendo que os animais considerados puros possuem uma VM menor em relação aos animais sintéticos. Entretanto, touros da raça Brangus apresentaram maior VM quando comparados com outras raças (BARCA Jr. et al., 2018). No presente estudo ocorreu uma correlação negativa entre a espessura de pele e IR.

Apesar do IR não ter sido diferente entre os formatos testiculares dos animais avaliados, o IR e o IP foram numericamente maiores em animais MO comparados com LO. Barca Jr. (2017) avaliaram touros de diversas raças e obtiveram resultados semelhantes em touros com média de 24 meses da raça Brangus, porém apresentaram um valor menor de IR $(0,36)$ e IP $(0,30)$. Além disso, trabalhos em outras espécies determinaram que os elevados valores de RI como em humanos, garanhões, cães e camelídeos estão associados à infertilidade (BIAGIOTTI et al., 2002; POZOR; MCDONNELL, 2002; PINGGERA et al., 2008; KUTZLER et al.,2011; ZELLI et al., 2013).

Neste estudo, o PE médio dos animais foi de $32,8 \mathrm{~cm}$, e isso pode ser explicado pelo fato dos animais se encontrarem em regime alimentar diferenciado levando a um maior desenvolvimento das medidas corporais e do PE (COULTER et al., 1987; BOLIGON et al., 2010). Similarmente, Coulter (1991) sugeriu que animais entre 12 a 14 meses devem apresentar PE de 32,0 cm para raça Aberdeen Angus, sendo que esses valores entre os 15 a 20 meses de idade se alteram para $34 \mathrm{~cm}$. A idade dos animais exerce grande efeito sobre o desenvolvimento testicular em machos de 6 a 36 meses, sendo que o maior crescimento testicular ocorre entre 6 e 16 meses de idade, tendo diferença de tamanho entre as raças (COULTER, 1991). O desenvolvimento testicular altera drasticamente com a idade dos animais, em touros ocorre um crescimento retardado dos testículos antes dos 6 meses de idade e um crescimento rápido até a puberdade (RAWLING et al., 2008). Menegassi et al. (2019) observaram que o PE ajustado em reprodutores entre 16 e 18 meses apresentaram maior taxa de crescimento do PE em animais B. taurus indicus e cruzas, Braford e Brangus, em comparação com raças Europeias. Portanto, o PE tem importância por ser um indicador do volume testicular e ter alta correlação com a produção espermática, sendo utilizado como parâmetro de seleção de touros jovens em função de sua facilidade de mensuração e precisão.

Conforme a idade dos animais aumenta, a pele aumenta de espessura por diversas razões e isso pode ter influência na termorregulação, sendo necessário um aprofundamento nas pesquisas nesta área. A medida de PE é, sem dúvidas, um indicador útil do tamanho testicular, da capacidade de produção espermática e da idade à puberdade, tanto nos machos, como nas fêmeas aparentadas, principalmente meio-irmãs e filhas desses machos (BRINKS et al.,1978; COULTER; KELLER, 1982; GRESSLER et al., 2000).

De acordo com Ellis et al. (2005), animais de 12 a 24 meses de idade estão em progressiva maturação sexual e são mais sensíveis e vulneráveis a fatores que influenciam a fertilidade e variabilidade no desempenho reprodutivo. Fatores como estação do ano, alterações nas temperaturas climáticas, lesões e estresse fisiológico foram relacionados com alterações na qualidade seminal. Também sob a ação de ITU médio de $(74,5)$, muito próximo a zona de estresse térmico, acarreta alterações do fluxo sanguíneo testicular, influenciados pela presença de termorreceptores no escroto, responsáveis por enviar sinais que estimulam uma resposta fisiológica à dissipação de calor para manter a temperatura escrotal (NANAYAKKARA, 2011). Vale ressaltar que Silva et al. (2017) observaram que os búfalos são eficientes para dissipar a energia térmica, mesmo em níveis de ITU altos, não prejudicando a termorregulação testicular e escrotal.

\section{Conclusão}

As condições climáticas, determinadas por ITU, não foram suficientes para alterar drasticamente os valores da hemodinâmica testicular em função do reflexo fisiológico da termorregulação escrotal. Animais com formato testicular considerado moderado oval apresentaram maior resistência vascular imposta ao fluxo sanguíneo pela diferença anatômica na artéria supra testicular, indicando melhor dissipação do calor neste formato. Em função da forma mais alongada dos testículos de animais pré-púberes da raça Brangus, esses parâmetros associados aos valores de hemodinâmica testicular servirão para avaliar com maior precisão o potencial do futuro reprodutor.

\section{Referências}

AZEVEDO, M. et al. Estimativas de níveis críticos superiores do índice de temperatura e umidade para vacas leiteiras $1 / 2,3 / 4 \mathrm{e}$ 7/8 Holandes-Zebu em lactação. Rev. Bras. Zootec., v.34, p.20002008, 2005. doi: 10.1590/S1516-35982005000600025.

Bailey, T.L. et al. Testicular shape and its relationship to sperm production in mature Holstein bulls. Theriogenology, v.46, p.881887, 1996. doi: 10.1016/s0093-691x(96)00245-2.

Bailey, T.L. et al. Caliper and ultrasonographic measurements of bovine testicles and a mathematical formula for determining testicular volume and weight in vivo. Theriogenology, v.49, p.581-594,1998. doi:10.1016/s0093-691x(98)00009-0.

Barca Junior, F.A. et al. Effect of breed on testicular blood flow dynamics in bulls. Theriogenology, v.118, p.16-21, 2018. doi: 
10.1016/j.theriogenology.2018.05.022

Biagiotti, G. et al. Spermatogenesis and spectral echo-colour Doppler traces from the main testicular artery. BJU Int., v.90, p.903-908, 2002. doi: 10.1046/j.1464-410x.2002.03033.x

Boligon, A.A. et al. Estimation of genetic parameters for body weights, scrotal circumference, and testicular volume measured at different ages in Nellore cattle. J. Anim. Scie., v.88, p.12151219, 2010. doi: 10.2527/jas.2008-1719

Brinks, J.S. et al. Relationship of age at puberty in heifers to reproductive traits in young bulls. Proc. Western Section Am. Soc. Anim. Scie., v.29, p.28-30, 1978.

Brito, L.F. et al. Effects of scrotal insulation on sperm production, semen quality, and testicular echotexture in Bos indicus and Bos indicus x Bos taurus bulls. Anim. Reprod. Scie., v.79, p.1-15. 2003. doi: 10.1016/s0378-4320(03)00082-4.

Brito, L.F. et al. Testicular thermoregulation in Bos indicus, crossbred and Bos taurus bulls: relationship with scrotal, testicular vascular cone and testicular morphology, and effects on semen quality and sperm production. Theriogenology, v.61, p.511-528, 2004. doi:10.1016/s0093-691x(03)00231-0.

Carrillo, J.D. et al. Colour and pulsed Doppler ultrasonographic study of the canine testis. Reprod. Domestic Anim., v.47, p.655659, 2012. doi: 10.1111/j.1439-0531.2011.01937.x

Claus, L.A.M. et al. Scrotal skin thickness, testicular shape and vascular perfusion using Doppler ultrasonography in bulls. Livestock Scie., v.226, p.61-65, 2019. doi: 10.1016/j. livsci.2019.06.005

Corbet, N.J. et al. Male traits and herd reproductive capability in tropical beef cattle. 2. Genetic parameters of bull traits. Anim. Prod. Scie., v.53, p.101-113, 2013. doi: 10.1071/AN12163

Coulter, G.H.; FOOTE, R.H. Bovine testicular measurements as indicators of reproductive performance and their relationship to productive traits in cattle: a review. Theriogenology, v.11, p.297311, 1979. doi: 10.1016/0093-691x(79)90072-4

Coulter, G.H; KELLER, D.G. Scrotal circumference of young beef bulls; relationship to paired testes weight effect to breed, and predictability. Canadian J. Anim. Scie., v.62, p.133-139, 1982. doi: $10.4141 /$ cjas $82-013$

Coulter, G.H. et al. Scrotal circumference of two-year-old bulls of several beef breeds. Theriogenology, v.27, p.485-491, 1987. doi: 10.1016/0093-691x(87)90236-6

Coulter, G.H. Scrotal circumference: a review. Proc. Soc. Theriogenol., p.113-116, 1991.

De Brito, M. et al. Doppler and contrast enhanced ultrasonography of testicles in adult domestic felines. Reprod. Domestic Anim., v.50, p.730-734, 2015. doi: 10.1111/rda.12557

Dowling, D. F. The significance of the thickness of cattle skin. J. Agricul. Scie., v.62, p.307-311, 1964. doi: 10.1017/ S0021859600042398

Ellis, R. W. et al. Fertility of yearling beef bulls during mating. Theriogenology, v.64, p.657-678, 2005. doi: 10.1016/j. theriogenology.2005.05.029

Fields, M. J.; Burns, W. C.; Warnick, A.C. Age, season and breed effects on testicular volume and semen traits in young beef bulls. J. Anim. Scie., v.48, p.1299-1304, 1979. doi: 10.2527/ jas1979.4861299x

Galina, C.S.; Horn, M.M.; Molina, R. Reproductive behaviour in bulls raised under tropical and subtropical conditions. Hormones and Behaviour, v.52, p.26-31, 2007. doi: 10.1016/j. yhbeh.2007.03.026
Gerhard-Herman, M. et al. Guidelines for noninvasive vascular laboratory testing: a report from the american society of echocardiography and the society for vascular medicine and biology. Vascular Med., v.11, p.183-200, 2006. doi: $10.1177 \% 2 \mathrm{~F} 1358863 \times 06070516$

Gressler, S.L. et al. Estudo das associações genéticas entre perímetro escrotal e características reprodutivas de fêmeas Nelore. Rev. Bras. Zootec., v.29, p.427-437, 2000. doi: 10.1590/ S1516-35982000000200016

Herzog, K.; Bollwein, H. Application of doppler ultrasonography in cattle reproduction. Reprod. Domestic Anim., v.42, p.51-58, 2007. doi: 10.1111/j.1439-0531.2007.00903.x

IBGE - Instituto Brasileiro de Geografia e Estatística. Cidades@. Brasília: IBGE, 2016.

Instrutherm. 2010. Manual de instruções: medidor de stress térmico modelo TGD-400. São Paulo: Instrutherm.

Jian, W. et al. Differences of skin morphology in Bos indicus, Bos taurus, and their crossbreds. Int. J. Biometeorol., v.58, p.10871094, 2014. doi: 10.1007/s00484-013-0700-9

Kastelic, J.P. Scrotal/testicular thermoregulation and the effects of testicular temperature in the bull. Vet. Clin. North Am. Food Anim. Pratice, v.13, p.271-282, 1997. doi: 10.1016/s07490720(15)30340-6

Kastelic, J.P. Understanding and evaluating bovine testes. Theriogenology, v.81, p.18-23, 2014. doi: 10.1016/j. theriogenology.2013.09.001

Kutzler, M. et al. Determination of testicular blood flow in camelids using vascular casting and color pulsed-wave doppler ultrasonography. Vet. Med. Int., 2011. doi: 10.4061/2011/638602

$\mathrm{Li}, \mathrm{S}$. et al. Evaluation of thermal stress indices for cattle. In American Society of Agricultural and Biological Engineers Annual International Meeting, v.4, p.2283-2302, 2009.

Matton, J. S.; Nyland, T. G. Próstata e testículo. In: NYLAND, T.G.; MATTON, J.S. Ultrassom: diagnóstico em pequenos animais. São Paulo: Rocca. p.255-271, 2004.

Menegassi, S.R.O. et al. Evaluation and prediction of scrotal circumference in beef bulls. Theriogenology, v.140, p.25-32, 2019. doi: 10.1016/j.theriogenology.2019.08.008

Menegassi, S. R. O. et al. Scrotal infrared digital thermography as a predictor of seasonal effects on sperm traits in Braford bulls. Int. J. Biometeorol., v.59, p.357-364, 2014. doi: 10.1007/s00484014-0847-z

Menegassi, S. R. O. et al. Effects of ambient air temperature, humidity, and wind speed on seminal traits in Braford and Nellore bulls at the Brazilian Pantanal. Int. J. Biometeorol., v.60, p.17871791, 2016. doi: 10.1007/s00484-016-1167-2

Middleton, W.D.; Thorne, D.A.; Melson, G.L. Color Doppler ultrasound of the normal testis. Am. J. Roentgenol., v.152, p.293297, 1989. doi: 10.2214/ajr.152.2.293

Nanayakkara, S.D.I. The physiological mechanisms of scrotal temperature regulation and the effects of its dysregulation on endocrine function of the testes. University of Western Australia. 2011

Patil, V.; Shetty, S. M.; Das, S. Common and uncommon presentation of fluid within the scrotal spaces. Ultrasound Int. Open, v.1, p.34-40, 2015. doi: 10.1055/s-0035-1555919

Pinggera, G. M. et al. Assesement of the intratesticular resistive index by color Doppler ultrasonography measurements as a predictor of spermatogenesis. BJU Int., v.101, p.722-726, 2008. doi: 10.1111/j.1464-410X.2007.07343.x 
Pozor, M.A.; MCDONNELL, S.M. Doppler ultrasound measures of testicular blood flow in stallions. Theriogenology, v.584, p.3740, 2002. doi: 10.1016/s0093-691x(03)00227-9

POZOR, M.A.; MCDONNELL, S.M. Color Doppler ultrasound evaluation of testicular blood flow in stallions. Theriogenology, v.61, p.799-810, 2004. doi: 10.1016/s0093-691x(03)00227-9

Pugliesi, G. et al. Use of color-Doppler ultrasonography for selection of recipients in timed-embryo transfer programs in beef cattle. Theriogenology, v.135, p.73-79, 2019. doi: 10.1016/j. theriogenology.2019.06.006

R Core Team. R: A language and environment for statistical computing. R Foundation for Statistical Computing, Vienna, Austria, 2014.

Ravagnolo, O.; Misztal, I.; Hoogenboom, G. Genetic component of heat stress in cattle, development of a heat index function. J. Dairy Scie., v.83, p.2120-2125, 2000. doi: 10.3168/jds.S00220302(00)75094-6

Rawlings, N.C. et al. Sexual maturation in the bull. Supplement Reproduction in Domestic Animals, v.295, p.1, 2008. doi: 10.1111/j.1439-0531. 2008.01177.x.

Sanyal, D.C.; Maji, N. K. Thermoregulation through skin under variable atmospheric and physiological conditions. J. Theor.
Biol., v.208, p.451-456, 2001. doi: 10.1006/jtbi.2000.2231

Silva, L.K.X. et al. Testicular thermoregulation, scrotal surface temperature patterns and semen quality of water buffalo bulls reared in a tropical climate. Andrologia, v.50, p.1-10, 2017. doi: 10.1111/and.12836

Siqueira, J. B. et al. Testicular shape and andrological aspects of young Nellore bulls under extensive farming. Rev Bras. Zoote., v.41, p.612-617, 2012. doi: 10.1590/S1516-35982012000300020

Smith, B. A.; Brinks, J.S.; Richardson, G.V. Relationships of sire scrotal circumference to offspring reproduction and growth. J. Anim. Scie., v.67, p.2881-2885, 1989. doi: 10.2527/ jas1989.67112881x

Thom, E. C. The discomfort index. Weatherwise, v.12, p.57-59, 1959. doi: $10.1080 / 00431672.1959 .9926960$

Viu, M. A. O. et al. Testicular biometry, semen quality and sexual maturity of Nellore young bulls raised in the Brazilian tropical savannah. J. Anim. Vet. Adv., v.14, p.144-150, 2015.

Wildeus, S.; Holroyd, R.G.; Entwistle, K. W. Patterns of pubertal development in Sahiwal and Brahman cross bulls in tropical Australia. I. Growth and semen characteristics. Theriogenology, v.22, p.361-373, 1984. doi: 10.1016/0093-691X(84)90456-4. 